\title{
Dynamometric Indicators of Fatigue From Repeated Maximal Concentric Isokinetic Plantar Flexion Contractions Are Independent of Knee Flexion Angles ANd Age but Differ for Males and Females
}

\author{
Kim Hébert-Losier and Hans-Christer Holmberg \\ Department of Health Sciences, Swedish Winter Sports Research Centre, Mid Sweden University, Östersund, Sweden
}

\begin{abstract}
Hébert-Losier, K and Holmberg, HC. Dynamometric indicators of fatigue from repeated maximal concentric isokinetic plantar flexion contractions are independent of knee flexion angles and age but differ for males and females. J Strength Cond Res 28 (3): 843-855, 2014-Sex and age are reported to influence the maximal dynamometric performance of major muscle groups, inclusive of ankle plantar flexors. Knee flexion (KF) also impacts plantar flexion function from where stems use of $0^{\circ}$ and $45^{\circ}$ angles of $\mathrm{KF}$ for clinical assessment of gastrocnemius and soleus, respectively. The influence of KF, sex, and age on dynamometric indicators of plantar flexion fatigue was examined in 28 males and 28 females recruited in 2 different age groups (older and younger than 40 years). Each subject performed 50 maximal concentric isokinetic plantar flexions at 60-degree angle per second with $0^{\circ}$ and $45^{\circ}$ angles of KF. Maximal voluntary isometric contractions were determined before and after isokinetic trials and maximal, minimal, and normalized linear slopes of peak power during testing. Main effects of and 2way interactions between KF, sex, age, and order of testing were explored using mixed-effect models and stepwise regressions. At angles of $0^{\circ}$ and $45^{\circ}$, the fatigue indicators in younger and older individuals were similar and not influenced by testing order. However, peak isokinetic power and isometric torque declined to greater extents in males than females and, moreover, KF exerted greater impacts on the absolute plantar flexion performance and maximal-to-minimal reduction in isokinetic power in males. Because KF wielded no pronounced effect on fatigue indicators, this test may perhaps be used over time with no major concern regarding the exact knee angle. Our
\end{abstract}

Address correspondence to Kim Hébert-Losierkim, kim.hebert-losier@ miun.se.

28(3)/843-855

Journal of Strength and Conditioning Research

(C) 2014 National Strength and Conditioning Association findings indicate that sex, rather than age, should be considered when interpreting dynamometric indicators of fatigue from repeated maximal concentric isokinetic plantar flexions, for example, when establishing normative values or comparing outcomes.

KEY WoRDs ankle, dynamometer, endurance, fatigability, lower extremity, triceps surae muscle

\section{INTRODUCTION}

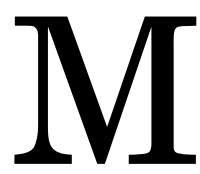

uscle endurance is an important aspect of strength and conditioning, and for the prevention of musculoskeletal injuries (14). Accordingly, the ability of muscles to resist fatigue is assessed in clinical and athletic contexts. Endurance of the main ankle plantar flexors-the triceps surae muscles-is essential for optimal performance and response to repeated loading (e.g. walking, running, and jumping) (24). In clinical environments, manual and dynamometric procedures are frequently employed to evaluate and train muscle function, using various knee flexion $(\mathrm{KF})$ angles to assess the relative contributions of the soleus and gastrocnemius muscles to plantar flexion (29).

One of the most common manual endurance testing procedures for ankle plantar flexion involves performing a maximal number of unilateral heel raise cycles (25) at different $\mathrm{KF}$ angles. However, this maximal number with angles of $0^{\circ}, 30^{\circ}$, and $45^{\circ}$ of $\mathrm{KF}$ has recently been reported to be similar by 2 separate research studies $(22,23)$, with no marked differences in the surface electromyography signals from either the gastrocnemius or soleus muscles at angles of $0^{\circ}$ and $45^{\circ}$ of $\mathrm{KF}$. Thus, the clinical utility and validity of performing the heel-raise test at different $\mathrm{KF}$ angles must be questioned and alternative procedures be sought.

Dynamometry provides more objective quantification than manual muscle testing and is more sensitive to alterations in performance (20). For several decades, isokinetic dynamometry has been used effectively in clinics and 
research for muscle assessment and rehabilitation. Evaluation of the function of the triceps surae muscles after injury, surgery, and neuropathy with this approach is documented $(1,2)$ and reported to indicate the frequency and severity of muscle weakness more precisely than manual muscle testing (2), which mistakenly classified the actual strength of the plantar flexor muscles in $41 \%$ of patients with neuropathies. However, it is not yet clear whether the KF angles recommended in clinical environments for training and evaluation of the soleus ( $45^{\circ}$ angle) and gastrocnemius $\left(0^{\circ}\right.$ angle) muscles influence the dynamometric indicators of fatigue caused by successive isokinetic plantar flexion contractions.

There are several reports on prolonged plantar flexion isometric contractions at various degrees of $\mathrm{KF}$, but only a few on repeated isokinetic contractions. In comparing performance of 50 isokinetic contractions at angles of $0^{\circ}, 45^{\circ}$, and $90^{\circ}$ of KF, Signorile et al. (43) employed electromyographic measurements, which are often impossible or less practical for clinicians to perform than dynamometric measurements alone. Among other information, dynamometric indicators of plantar flexion fatigue reveal reductions in mean and/or peak torque, work and power that occur during or immediately after an exhaustive task.

Recently, Harbo et al. (20) established normative data for the dynamometric assessment of major muscle groups, inclusive of the ankle plantar flexors that include predictors of performance based on sex and age. In their study, males were consistently stronger than females, and dynamometric performance was inversely related to age. These recently documented sex differences are in agreement with those reported previously by the research teams of Trappe et al. (44) and Danneskiold-Samsøe et al. (13), who found plantar flexion torque to be $40-50 \%$ higher in males than females, as expected from their relative statures and muscle masses (27). In older individuals, Morse et al. (36) and Gajdosik et al. (18) also observed reductions in peak isometric and isokinetic plantar flexion performance that were associated with alterations in the cross-sectional area and contractile properties of muscles.

Although these studies provide insight on plantar flexion dynamometric strength profiles, they provide no information pertaining to the influence of $\mathrm{KF}$, sex, and age on the fatigability of the triceps surae muscles during dynamometric assessment. One study by Larsson et al. (31) did examine the performance of 100 maximal concentric isokinetic plantar flexion contractions in 9 males and 43 females in their mid-twenties. These authors reported a more pronounced and rapid decline in plantar flexion output in males that correlated with larger and higher proportion of type II (fast-twitch) muscle fibers in the triceps surae. However, because this study did not involve a balanced number of males and females or individuals older than 30 years, it is uncertain whether their findings can be applied to the general population. Deriving data on plantar flexion fatigability in a range of healthy adults would assist in further under- standing typical triceps surae muscle function and clarify which factors should be considered during assessment of plantar flexion endurance using isokinetic dynamometry.

Accordingly, the present study was designed to characterize the influence of $\mathrm{KF}$ angle, sex, and age on the dynamometric indicators of plantar flexion fatigue from repeated maximal concentric isokinetic contractions. In light of the fact that overall triceps surae muscle activity tends to fall with increasing $\mathrm{KF}$ angle (3) and the empirical findings described above, our hypotheses were that a greater $\mathrm{KF}$ angle, an older age, and the male sex would be associated with outcomes indicating more pronounced plantar flexion fatigue.

\section{Methods}

\section{Experimental Approach to the Problem}

The influence of $\mathrm{KF}$ angle, sex, and age on the dynamometric indicators of plantar flexion fatigue was examined employing a repeated-measures design that required each subject to participate in 1 experimental session, that included familiarization, at the Isokinetic Laboratory of the Swedish Winter Sports Research Center. Each subject was tested at angles of $0^{\circ}$ and $45^{\circ}$ of $\mathrm{KF}$ to investigate the effect of $\mathrm{KF}$ angle. Although $90^{\circ}$ angle of $\mathrm{KF}$ would have enhanced the likelihood of detecting differences in plantar flexion performance (5), $45^{\circ}$ angle was chosen because it is routinely recommended for testing and training of the soleus muscle $(1,29)$ and, moreover, influences the function of the triceps surae muscles and plantar flexion output in a manner that is distinct from $0^{\circ}$ angle $(3,43)$. In addition, the recruitment process targeted an equal number of males and females to investigate to effect of sex on outcomes, dichotomized in younger ( $20-40$ years) and older ( $40-60$ years) age groups to explore the effect of age. The age of 40 years was selected to dichotomize age considering that strength and muscle mass peaks around 25 years of age remains relatively stable until 35 or 40 years and then shows an accelerating decline (42), with deteriorations in strength, balance, and endurance noted at 40 years (16). In summary, the dependent variables were the dynamometric indicators of plantar flexion fatigue (explained below), and the dichotomized independent variables were the $2 \mathrm{KF}$ angles $\left(0^{\circ}\right.$ and $\left.45^{\circ}\right)$, sexes (male and female) and age groups (20-40 years and 40-60 years). Because all testing was performed in a single session, fatigue from the first test might have affected performance in the second test despite planned rest periods, so the order of testing (first and second) was also included as an independent variable.

\section{Subjects}

The subjects in this study were volunteers from the local community who were recruited using advertisements in e-mail distribution lists, local newspapers, bulletin boards, online forums, and word-of-mouth. After providing written informed consent, 56 volunteers selected on the basis of sex 
and age completed this study. Half of the subjects were males (mean $\pm S D$; age: $38.9 \pm 11.3$ years; height: $181 \pm$ $7 \mathrm{~cm}$; mass: $81.5 \pm 8.9 \mathrm{~kg}$; body mass index: $24.9 \pm 2.3$ $\mathrm{kg} \cdot \mathrm{m}^{-2}$ ) and the other half were females (age: $40.8 \pm 11.3$ years; height: $169 \pm 8 \mathrm{~cm}$; mass: $68.2 \pm 8.1 \mathrm{~kg}$; body mass index: $23.8 \pm 2.7 \mathrm{~kg} \cdot \mathrm{m}^{-2}$ ), with equal numbers of individuals between $20-40$ and $40-60$ years of age. The age of each subject was determined from the exact date of birth and date of testing, with no subject tested on their exact date of birth.

The inclusion criteria were good self-reported general health with regular participation in recreational physical activity and no current or recent ( $<3$ months) musculoskeletal injuries, joint pathologies, or other medical conditions that could limit performance of repeated maximal concentric isokinetic plantar flexion contractions. The exclusion criteria were previous experience with the isokinetic device for muscle testing or training, participation in highly competitive or elite-level sports and, in women, the possibility of pregnancy. Unless they were competitive bodybuilders or weightlifters, individuals who performed weight/resistance training were not excluded, and no inclusion/exclusion criteria were formulated on the basis of estrogen levels, menstrual cycles, and hormone replacement therapies. The research protocol was preapproved by the Regional Ethical Review Board (Umeå, Sweden, 2012-99-32M) and adhered to the Declaration of Helsinki, with appropriate informed consent obtained from all subjects. All testing was completed within early spring of the same year.

\section{Procedures}

In a single session at the muscle performance laboratory of our university sports research facility, the subjects were familiarized with the experimental protocol and then tested. To minimize intertester variability, the same experienced investigator conducted all testing. The IsoMed2000-dynamometer (D\&R Ferstl GmbH, Hemau, DE) used was calibrated by the manufacturer, and this calibration was verified in-house each day before data collection. The Dunedin Footedness Inventory involves kicking a ball, stamping out a simulated fire, picking up a marble, and tracing a shape with the lower extremity (41) and was used to determine foot dominance, revealing right foot dominance in 50 of the subjects and left foot dominance in the remaining 6. The short version of the International Physical Activity Questionnaire (11) was also administered and showed high, moderate, and low levels of physical activity in 24,20 , and 12 subjects, respectively. These levels were distributed equally amongst males and females older than and younger than 40 years (Kruskal-Wallis test, $p=0.873$ ), allowing for valid comparisons between age and sex with little bias from physical activity levels.

To eliminate the potential influence of testing order and fatigue, each subject was tested at angles of $0^{\circ}$ and $45^{\circ}$ of $\mathrm{KF}$ in a block randomized order according to age and sex, with half of the males and half of the females in each age group beginning at $0^{\circ}$ angle and the others at $45^{\circ}$ angle of KF. This randomization ensured that any effect of fatigue from the first test on the second test would be the same for all dependent variables.

\section{Experimental Setup}

Although placed in supine on the IsoMed2000-dynamometer, the dominant foot of the subject was attached firmly to the adapter using 3 Velcro straps: 1 on the distal aspect of the foot and 2 on its proximal aspect immediately distal to the ankle joint line (Figure 1A). The placement of the foot was adjusted to align the axes of rotation of the ankle joint and dynamometer, and 2 shoulder pads and a broad pelvic belt were used to minimize extraneous movements. The subject was also permitted to grasp the shoulder pads of the isokinetic device to enhance upper-body stabilization (Figure 1B).

Subsequently, the knee was fixed at an angles of either $0^{\circ}$ (knee straight with the femoral and tibial bones in alignment, Figure 1C) or $45^{\circ}$ (knee bent, Figure 1D). The investigator confirmed proper positioning using an extendable goniometer (Model01135, Lafayette Instrument, Loughborough, United Kingdom) following standard clinical guidelines and secured the knee angle with a thigh support. Stabilization of the knee and ankle was adjusted to prevent superior displacement and misalignment of the axes of rotation of the ankle and dynamometer during maximal plantar flexion efforts and verified before data collection.

Once the subject was positioned on the IsoMed2000dynamometer with angles of either $0^{\circ}$ or $45^{\circ}$ of $\mathrm{KF}$, the range of motion of the ankle was set for the isokinetic trials following a series of systematic steps (Figure 2). First, the ankle was placed in a neutral position (i.e. with the fifth metatarsal and fibula bones perpendicular to one another, as verified with the goniometer, Figure 2A). The foot adapter was thereafter moved passively to the end range of dorsi flexion (Figure 2B). From this position, the dynamometer was moved an angle of $5^{\circ}$ in the opposite direction toward plantar flexion to avoid strain from forceful muscle contraction in an elongated position. This ankle position defined the starting position of the concentric plantar flexion contractions (Figure 2B).

Thereafter, the foot adapter was moved actively to the end range of plantar flexion, stopping when the subject or investigator judged that the force of plantar flexion was no longer being effectively transferred to the foot adaptor. The dynamometer was then moved passively at an angle of $5^{\circ}$ in the opposite direction toward dorsi flexion to ensure effective force transmission and defined the end position of the concentric plantar flexion contractions (Figure 2C).

For the isokinetic trials, the IsoMed2000-dynamometer was programmed to monitor 50 consecutive concentric plantar flexion contractions at 60-degree angle per-second, with passive return of the ankle to the starting position at 90 degree angle per second. A total of 50 contractions were selected because, in trials involving 100 such contractions, torque has been reported to remain relatively stable after 50 

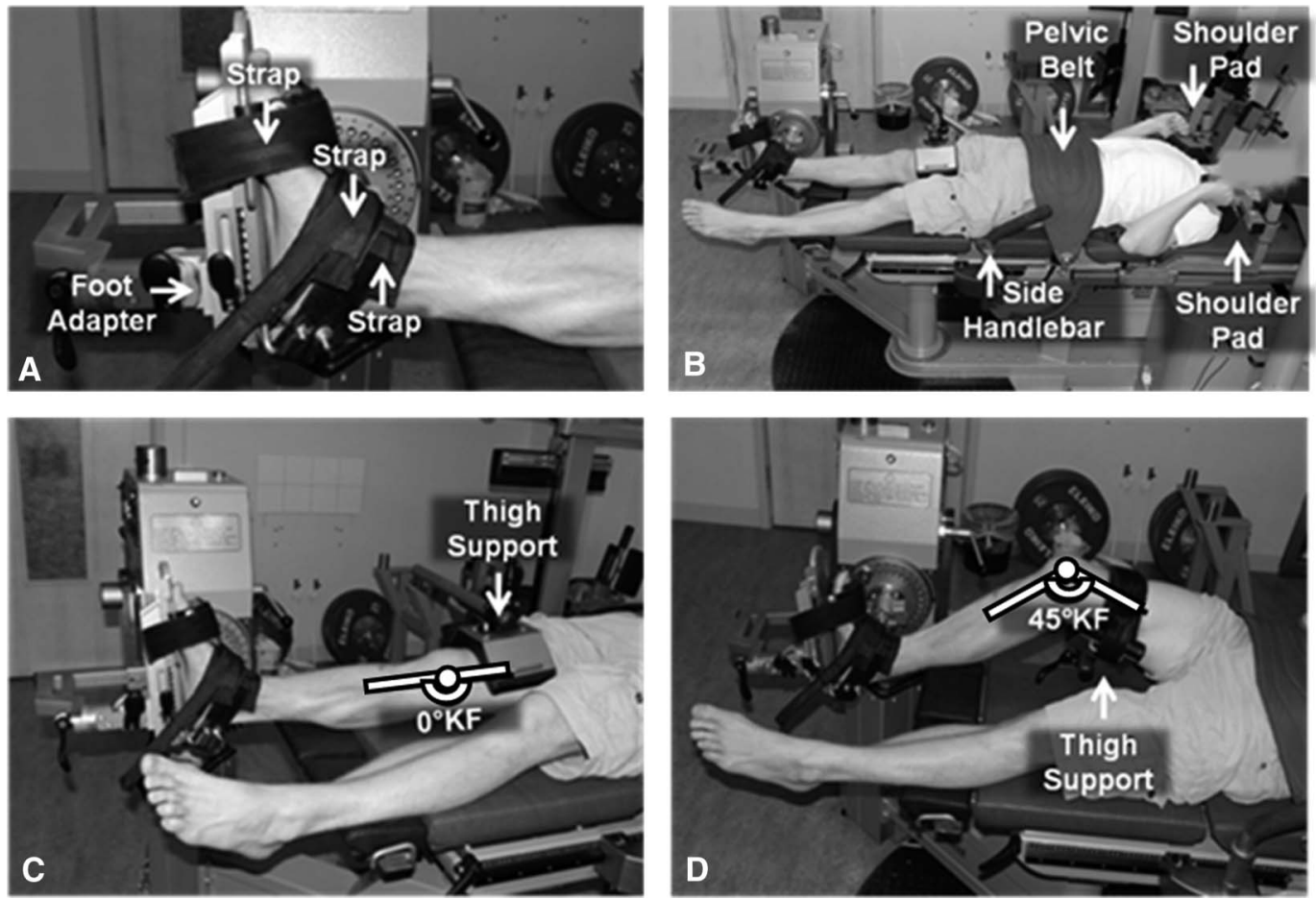

Figure 1. Positioning of the participant on the IsoMed2000-dynamometer. Knee flexion (KF) represents a joint angle. A) The dominant foot was secured to the adapter using 3 velcro straps. B) Shoulder pads, a pelvic belt, and side handlebars were used to minimize extraneous and upper-body motion. C) A support was placed above the thigh to secure the $\mathrm{KF}$ at $0^{\circ}$ angle. D) A support was placed below the thigh to secure the $\mathrm{KF}$ at $45^{\circ}$ angle.

contractions (32). Utilizing the velocity of 60 degree per second allowed comparison to prior isokinetic reports (31,32); whereas setting the passive return at angle 90 degree per second ensured a plantar flexion cycle of approximately $1 \mathrm{~Hz}$ (44). To optimize the isokinetic segment of the concentric plantar flexion contraction, rapid accelerations and decelerations were utilized, and to minimize force oscillations, $50 \mathrm{~N}$ was required to initiate each contraction.

\section{Experimental Protocol}

After determining height, body mass, and foot dominance, standard experimental procedures were followed (Figure 3), stating with positioning each subject on the IsoMed2000dynamometer at either $0^{\circ}$ or $45^{\circ}$ angle of $\mathrm{KF}$, and then setting the range of ankle motion for the isokinetic trial. The mean \pm $S D$ range of ankle motion was $44 \pm 11^{\circ}$ angle with the knee straight and $48 \pm 11^{\circ}$ angle with the knee bent, with paired

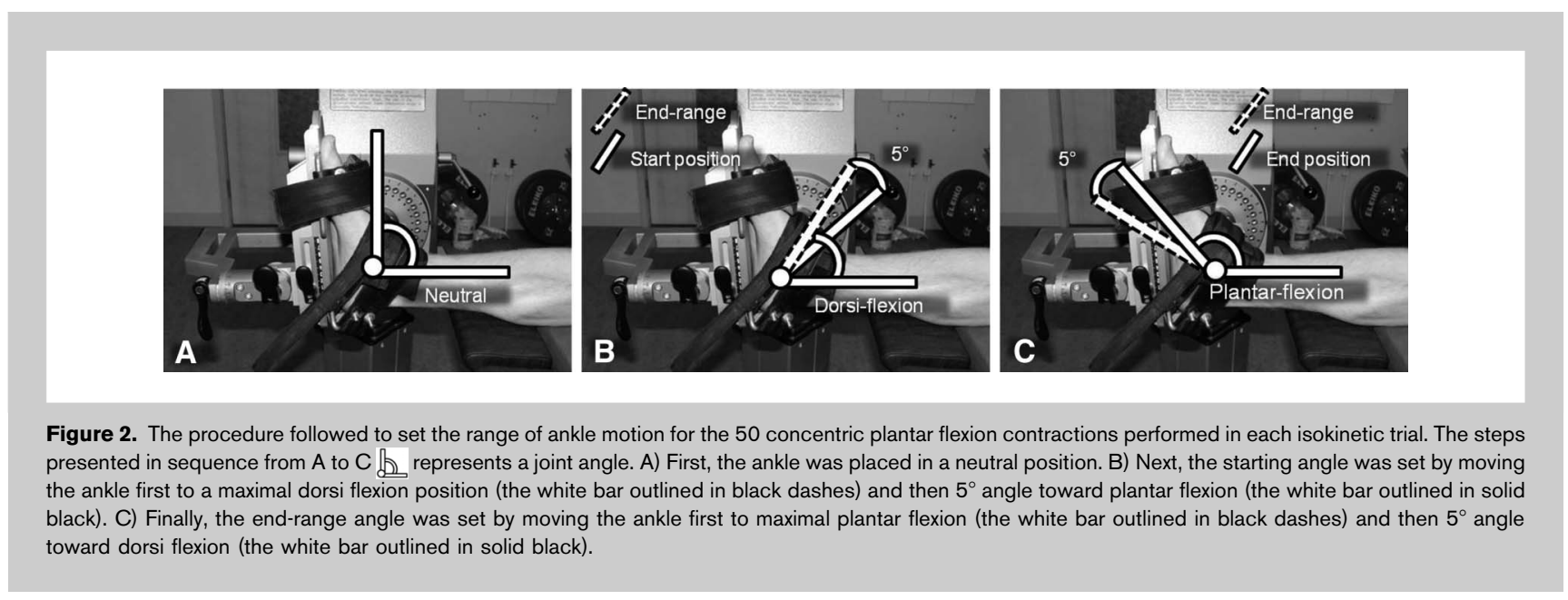




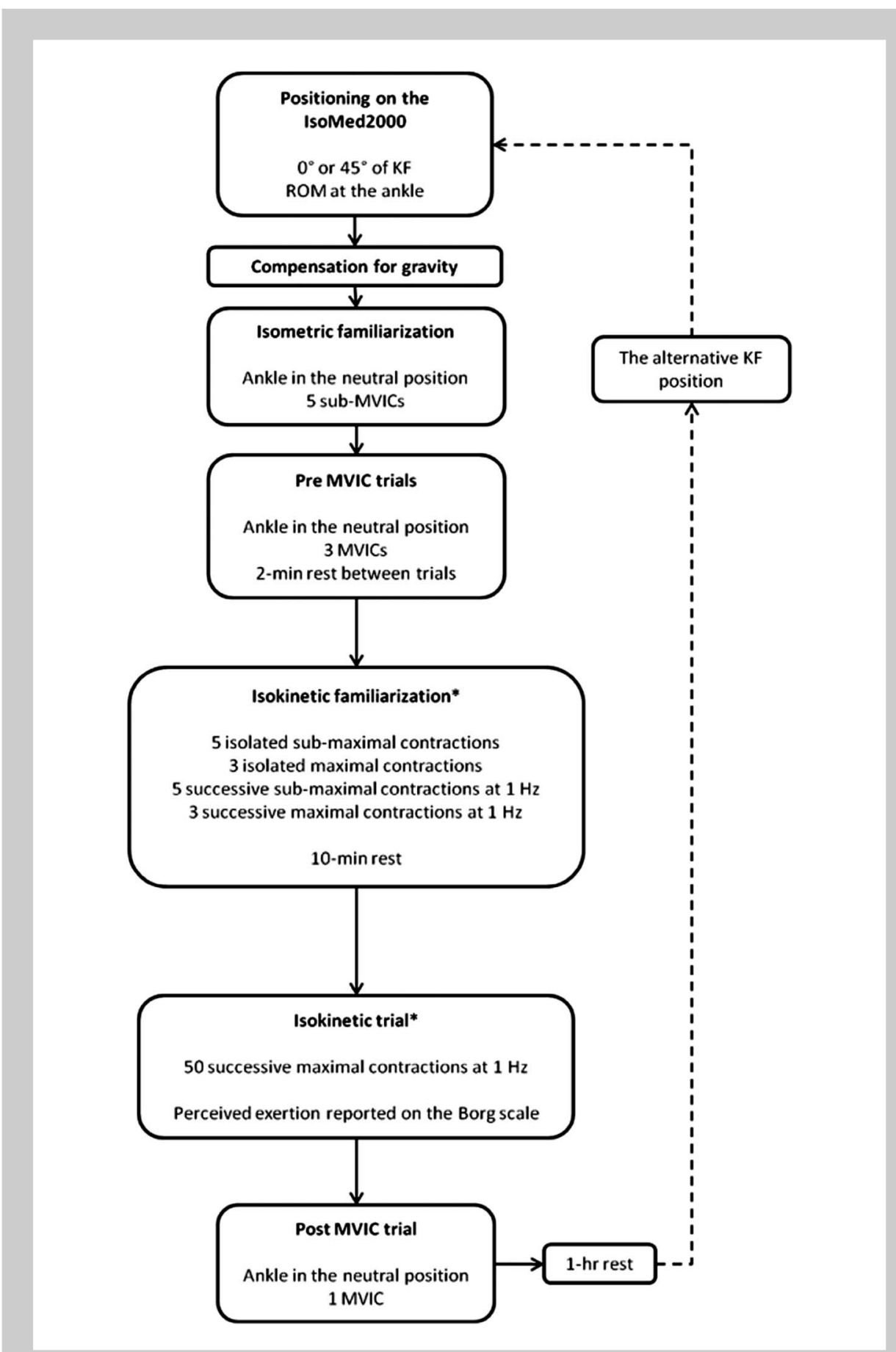

Figure 3. Flow diagram illustrating the experimental procedure for each subject. *Concentric plantar flexion contractions at $60^{\circ}$. angle per second with passive dorsi flexion return at $90^{\circ}$ angle per second through the set $\mathrm{ROM}$ of the ankle. $\mathrm{KF}=$ knee flexion; $\mathrm{MVIC}=$ maximal voluntary isometric contraction; $\mathrm{ROM}=$ range of motion.

gravity. Thereafter followed familiarization with maximal voluntary isometric contraction (MVIC) performance that involved 5 sub-MVICs, with 3 subsequent 7 -second MVICs (separated by 2 minutes of rest) to assess peak isometric torque.

During the subsequent warm-up and more specific familiarization, each subject practiced initiating and completing concentric isokinetic plantar flexion contractions at an angle of 60 degree per second throughout the permitted range of motion, relaxing as the dynamometer passively moved the ankle back to the starting position at an angle of 90 degree per-second. The isokinetic familiarization involved performing 5 isolated submaximal, 3 isolated maximal, 5 successive submaximal, and 3 successive maximal concentric plantar flexion contractions. The successive contractions were performed at a rate of $1 \mathrm{~Hz}$ that was indicated audibly to the subject by a computerized metronome (TempoPerfect v.2.02, $\mathrm{NCH}$ Software, Canberra, Australia). Each subject was then given a 10 -minute rest period to alleviate fatigue.

Before the actual isokinetic testing, each subject performed a single additional isolated 7second MVIC identical to the 3 that were performed previously to ensure that the peak torque remained within a $5 \%$ range (i.e., indicating no residual fatigue). The rest period was prolonged if the difference was greater than $5 \%$. None of the subjects expressed any residual fatigue or soreness from the procedures

$t$-testing indicating no significant differences between these 2 values $(p=0.078)$.

After setup on the dynamometer, the ankle was placed in the neutral position, and the subject was asked to remain as relaxed as possible during which time the integrated IsoMed2000 Software (v.2008.1.2RV1) compensated for preceding each isokinetic trial. Immediately before the isokinetic trial, each subject was reminded of the importance of pushing as hard as possible throughout the entire range of motion during each 1 of the 50 consecutive plantar flexion contractions, relaxing afterwards to allow passive return of the ankle to the starting position, and maintaining the $1 \mathrm{~Hz}$ pace. 
TABle 1. Mean $\pm S D$ and range (minimum-maximum) of the peak isometric torque associated with the maximal voluntary isometric contractions performed before (pre) and after (post) each isokinetic trial, in relationship to knee flexion $\left(0^{\circ}\right.$ and $\left.45^{\circ}\right)$, sex (male and female), and age (younger and older).*

\begin{tabular}{lcc}
\hline & \multicolumn{2}{c}{ Peak isometric torque $(\mathrm{N} \cdot \mathrm{m})$} \\
\cline { 2 - 3 } \multicolumn{1}{c}{ Variable } & \multicolumn{1}{c}{$0^{\circ}$ angle of KF } & $45^{\circ}$ angle of KF \\
\hline $\begin{array}{l}\text { Male }(n=28) \\
\quad \text { Pre }\end{array}$ & $222 \pm 54(151-333)$ & $272 \pm 73(136-425)$ \\
$\quad$ Post & $176 \pm 55(102-315)$ & $233 \pm 65(120-355)$ \\
Female $(n=28)$ & $154 \pm 36(96-240)$ & $176 \pm 54(92-323)$ \\
$\quad$ Pre & $127 \pm 39(55-197)$ & $148 \pm 47(61-248)$ \\
$\quad$ Post & $198 \pm 53(106-332)$ & $245 \pm 73(123-425)$ \\
Younger $(n=28)$ & $150 \pm 48(55-244)$ & $191 \pm 62(61-346)$ \\
$\quad$ Pre & & \\
$\quad$ Post & $179 \pm 60(96-333)$ & $203 \pm 81(92-379)$ \\
Older $(n=28)$ & $153 \pm 59(65-315)$ & $102 \pm 355(102-355)$ \\
$\quad$ Pre & Post &
\end{tabular}

*Younger $=20-40 \mathrm{y}$; older $=40-60 \mathrm{y} ; p \leq 0.001$ for $0^{\circ}$ angle vs. $45^{\circ}$ angle of KF; $P \leq 0.001$ for pre vs. post; $p \leq 0.001$ for male vs. female; $P=0.037$ for younger vs. older age; $\mathrm{KF}=$ knee flexion.

maximal and minimal peak torques during the 50 concentric isokinetic contractions were converted to units of power (torque $\times$ velocity, in watts [W]) to provide a measure specific to the isokinetic mode of testing and also subjected to analysis. Then, the line provided by the linear regression of the peak powers for all 50 isokinetic contractions was extrapolated to the $y$-axis. The normalized slope $\left(\% \cdot \mathrm{rep}^{-1}\right)$ of this line was used to define the rate of decline in peak power, with a more negative slope indicating a more rapid decrease in power.

In a separate study, the reproducibility of the dynamometric indicators of fatigue associated with our protocol was assessed in 34 males (age: $27.9 \pm 5.6$ years) in good gen-

The verbal introduction of "ready" and "3-2-1-PUSH" was provided to the subject as cue to initiate the 50 maximal isokinetic contractions.

Throughout the isokinetic trial, the investigator provided strong and consistent verbal encouragement, and the IsoMed2000 monitor displayed continuous visual feedback to promote maximal effort. The halfway point of $25 \mathrm{com}-$ pleted contractions was indicated to the subject, and the final 10 contractions were counted down. After completion, each subject rated the perceived exertion in their triceps surae muscles on the 6-20 points Borg scale. One final 7-second MVIC trial was then performed to assess the posttrial peak isometric torque. This post-MVIC was initiated $\sim 20$ seconds after the fatiguing task because the IsoMed2000-dynamometer does not allow a shorter interval. Because such delays may allow muscles to partially recover (45), the pre-to-post changes in MVIC torques may have been smaller with another dynamometer permitting shorter delays. Each subject then rested for 1 hour before repeating the procedure with the knee in the alternate position (Figure 3).

\section{Data Processing}

Angular position $\left({ }^{\circ}\right)$, velocity (degrees per-second), and torque $(\mathrm{N} \cdot \mathrm{m})$ data were transferred from the IsoMed2000 Software through a wireless connection to a computer; downloaded in the ASCII format; and processed with a fourth-order zero-lag Butterworth filter and cutoff frequency of $10 \mathrm{~Hz}$.

The peak isometric torque $(\mathrm{N} \cdot \mathrm{m})$ during the MVIC performed before and after the isokinetic trials at each of the $2 \mathrm{KF}$ angles was determined and retained for analysis. The eral health tested on 2 separate days 1 week apart. In this investigation, the pre-to-post change in peak isometric torque was associated with the largest typical error of measurement (coefficient of variation: 26\%), with corresponding values of $10-15 \%$ for measures relating to changes in peak isokinetic power during testing. These values are similar to others reporting the reproducibility of dynamometric indicators of fatigue at the ankle joint (39).

\section{Statistical Analyses}

The mean $\pm S D$ values of all variables were computed. Mixed-effects models and stepwise regressions were applied to assess the effects of $\mathrm{KF}$ (angles $0^{\circ}$ and $45^{\circ}$ ), sex (male and female), age (20-40 years and 40-60 years), and all possible 2-way interactions on plantar flexion performance. The model was also used to assess the main effect of (a) MVIC time (pre and post) on isometric torque, (b) isokinetic contraction (maximal and minimal) on peak power, and (c) order of testing (first and second) on isokinetic outcomes. Note that all potential 2-way interactions between the latter 2 variables (i.e. time and contraction) and the other factors (i.e. KF, sex and age) were also examined. Despite the measures taken to reduce order bias (e.g. block randomization and rest periods), the effect of order of testing was also considered because fatigue from the first test might have influenced performance during the second test. The model was based on a Gaussian (normal) distribution, clustered within-subject measures (random effects), and exchangeable correlation matrices. Because differences in the mass of male and female subjects might have influenced the relationships 


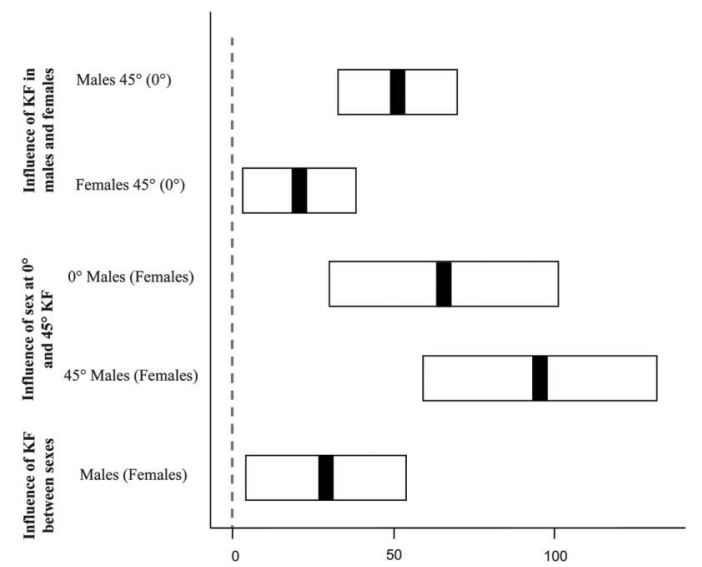

A

Difference in Peak Isometric Torque (Nm)
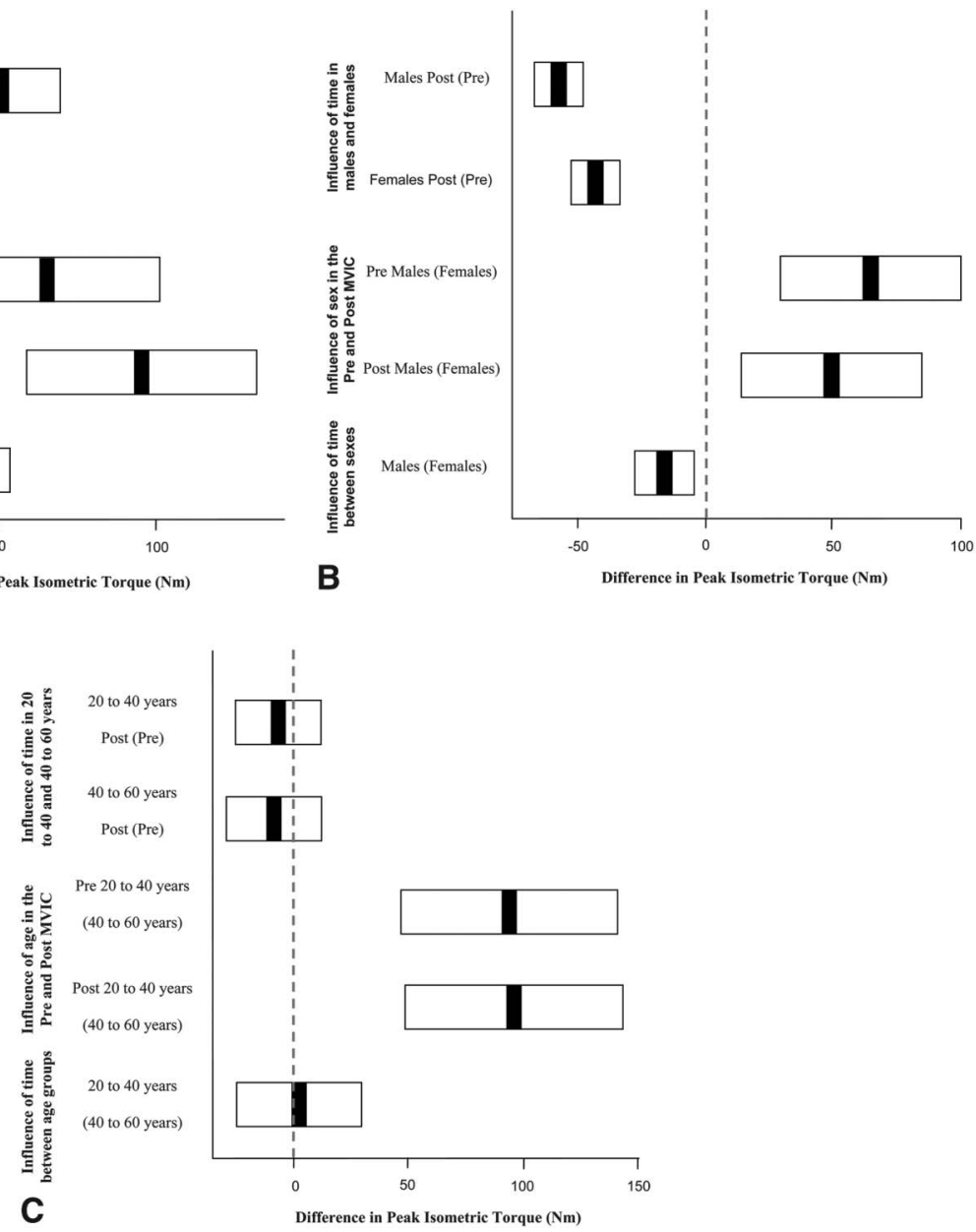

Figure 4. A) Differences in the peak isometric torque during a maximal voluntary isometric contraction (MVIC) in relationship to knee flexion (KF) and/or sex estimated employing a mixed-effects model. The reference value used for comparison is given in parentheses. The influence of KF between sexes is the change in torque at angles $0^{\circ}$ vs. $45^{\circ}$ of $\mathrm{KF}$ in one sex compared with the other. Comparison (reference), comparison minus reference; B) Differences in the peak isometric torque during an MVIC in relationship to the time at which the MVIC was performed and sex estimated employing a mixed-effects model. The reference value used for comparison is given in parentheses. The influence of the time at which MVIC was performed between sexes is the change in torque from the preto post-MVIC in one sex compared with the other. Comparison (reference), comparison minus reference; C) Differences in the peak isometric torque from an MVIC in relationship to the time at which the MVIC was performed and age group estimated employing a mixed-effects model. The reference value used for comparison is given in parentheses. The influence of the time at which the MVIC was performed between age groups is the change in torque from the pre to post MVIC in one age group compared with the other. Comparison (reference), comparison minus reference; represents mean; $\square$ represents $95 \%$ confidence interval; I represents no difference.

observed, when the effect of sex was significant, peak isometric torque $(\mathrm{N} \cdot \mathrm{m})$ and isokinetic power $(\mathrm{W})$ values were normalized to body mass and reanalyzed using the same approach to confirm these relationships.

Nonsignificant effects were removed sequentially from the original model using stepwise regression according to the Bayesian information criterion (8). Nonsignificant 2-way interactions were removed first, and the final model was thus composed solely of effects that significantly influenced the outcome. The coefficients from the final regression (expressed as $\beta[\mathrm{SE}]$ ) were used to estimate or compare outcomes obtained from testing under a known set of conditions. Effects that were not relevant to a given outcome were not considered during analysis, for example, the influence of isokinetic contraction (minimal, maximal) on the normalized slope of the line illustrating peak power, because all 50 contractions were used to compute this slope.

A value of $p \leq 0.05$ was considered to be statistically significant. Tukey Contrasts were employed for multiple comparisons of significant 2-way interactions, with Westfall adjustments for computation of the $95 \%$ confidence intervals [(CIs); lower, upper] and associated $p$-values (8). One-sided post hoc power analyses were performed on the dynamometric indicators of fatigue to determine whether our sample 
TABle 2. Mean $\pm S D$ and range (minimum-maximum) of the peak isokinetic power associated with the maximal (maximum) and minimal (minimum) $\left(0^{\circ}\right.$ and $\left.45^{\circ}\right)$, sex (male and female) and age (younger and older).*

\begin{tabular}{|c|c|c|}
\hline \multirow[b]{2}{*}{ Variables } & \multicolumn{2}{|c|}{ Peak isokinetic power (W) } \\
\hline & $0^{\circ}$ angle of $\mathrm{KF}$ & $45^{\circ}$ angle of $\mathrm{KF}$ \\
\hline \multicolumn{3}{|l|}{ Male $(n=28)$} \\
\hline Maximum & $145 \pm 34(94-240)$ & $164 \pm 41(70-237)$ \\
\hline Minimum & $60 \pm 12(24-81)$ & $75 \pm 24(42-137)$ \\
\hline \multicolumn{3}{|c|}{ Female $(n=28)$} \\
\hline Maximum & $107 \pm 32(57-188)$ & $113 \pm 38(58-195)$ \\
\hline Minimum & $51 \pm 16(29-88)$ & $51 \pm 13(29-82)$ \\
\hline \multicolumn{3}{|c|}{ Younger $(n=28)$} \\
\hline Maximum & $139 \pm 35(84-240)$ & $154 \pm 42(58-236)$ \\
\hline Minimum & $60 \pm 14(31-88)$ & $64 \pm 20(35-130)$ \\
\hline \multicolumn{3}{|c|}{ Older $(n=28)$} \\
\hline Maximum & $112 \pm 35(57-173)$ & $123 \pm 47(62-237)$ \\
\hline Minimum & $51 \pm 14(24-76)$ & $62 \pm 24(28-137)$ \\
\hline
\end{tabular}
contractions of the isokinetic plantar-flexion trials in relationship to knee flexion

$(p=0.0030$, Figure $4 \mathrm{~A})$, sex and the time at which the MVIC was performed $(p=0.0008$, Figure $4 \mathrm{~B})$, and age and the time at which the MVIC was performed $(p \leq 0.0001$, Figure 4C) remained significant.

Among others, between condition comparisons revealed that the difference in peak torques at angles of $0^{\circ}$ vs. $45^{\circ}$ of $\mathrm{KF}$ was greater in males than females $(30 \mathrm{~N} \cdot \mathrm{m}$ $[10,49], p=0.0021)$, as was the pre-to-post reductions in torque $(16 \mathrm{~N} \cdot \mathrm{m}[7,25], p \leq$ $0.0001)$, even when normalized to body weight. In contrast, the decline in torque from pre-topost MVIC trials was similar for the 2 age groups $(2 \mathrm{~N} \cdot \mathrm{m}$ $[-25,29], p=0.8351)$, as reflected in the overlapping means and 95\% CIs for the relevant values (Figure 4C).

size $(n=56)$ was large enough to detect a $20 \%$ change in mean values. All data processing and analyses took the repeated-measures design of this study into account and were carried out using R version 2.15.2 (R Foundation for Statistical Computing, Vienna, Austria) and Microsoft Excel 2010 (Microsoft Corp, Redmond, WA, USA).

\section{Results}

\section{Ratings of Perceived Exertion}

None of the 2-way interactions significantly influenced the ratings of perceived exertion, nor did sex or age alone $(p \geq$ $0.4274)$. However, the ratings of perceived exertion at the 2 $\mathrm{KF}$ angles $\left(18 \pm 1\right.$ [range: $15-20$ ] at $0^{\circ}$ and $17 \pm 2$ [range: $12-20]$ at $\left.45^{\circ}\right)$ differed significantly $\left(\beta_{\text {knee: }} 0.6[0.2], p \leq\right.$ $0.0001)$. A greater exertion was perceived after the second than the first test ( $\beta_{\text {order }}: 0.8[0.2], p \leq 0.0001$ ).

\section{Maximal Voluntary Isometric Contractions}

The peak MVIC torque values are summarized in Table 1 . For all trials combined, the pre-to-post change in torque was $18 \pm 13 \mathrm{~N} \cdot \mathrm{m}$, which gives an estimated power to detect a $20 \%$ variation in this mean of $70 \%$.

Stepwise regression eliminated the 2-way interaction between $\mathrm{KF}$ and time at which the MVIC was performed $(p=0.9031)$, indicating that the pre-to-post reductions in MVIC torque were similar at the $2 \mathrm{KF}$ angles. Likewise, the interaction between the order of testing and time of the MVIC was also removed, indicating that the decline in MVIC torque was similar during the first and second sessions of testing. On the other hand, the interactions between $\mathrm{KF}$ and sex
Considering the above interactions, the coefficients from the final regressed model provide the following estimates for the differences in peak torque values: $51[3] \mathrm{N} \cdot \mathrm{m}$ (3) lower during the MVIC performed after isokinetic test compared with before the isokinetic test $\left(\beta_{\text {time }}, p \leq 0.0001\right), 73$ [13] $\mathrm{N} \cdot \mathrm{m}$ lower in females than males $\left(\beta_{\mathrm{sex}}, p \leq 0.0001\right), 36[5]$ $\mathrm{N} \cdot \mathrm{m}$ lower with the knee at $0^{\circ}$ angle than $45^{\circ}$ angle $\left(\beta_{\text {knee, }} p \leq\right.$ $0.0001), 28[13] \mathrm{N} \cdot \mathrm{m}$ lower in older compared with younger individuals $\left(\beta_{\text {age, }} p=0.0375\right)$, and $12[5] \mathrm{N} \cdot \mathrm{m}$ lower in the second test compared with the first test ( $\left.\beta_{\text {order, }} p=0.0166\right)$.

\section{Isokinetic Trials}

The maximal, minimal, and normalized slope of the peak powers recorded during the isokinetic trials are documented in Table 2. For all isokinetic trials combined; the mean reduction in power from maximal-to-minimal contractions was $75 \pm 34 \mathrm{~W}$, whereas the reduction in power across the 50 contractions was $0.9 \pm 0.4 \%$. per repetition. Post hoc analyses revealed that study population was sufficiently large to detect a $20 \%$ change in these means with powers of $97 \%$ and $96 \%$, respectively.

Analysis of the normalized slopes revealed no significant influence of any of the 2-way interactions $(p \geq 0.1174)$ or of $\mathrm{KF}(p=0.2371)$, sex $(p=0.5184)$, age $(p=0.5568)$, or order of testing $(p=0.0916)$. Analysis of maximal and minimal peak powers yielded results similar to those obtained from analysis of pre-and post-MVIC torques. Stepwise regression eliminated the 2-way interactions between $\mathrm{KF}$ and isokinetic contraction $(p=0.3006)$, suggesting comparable differences between the maximal and minimal isokinetic contractions at 


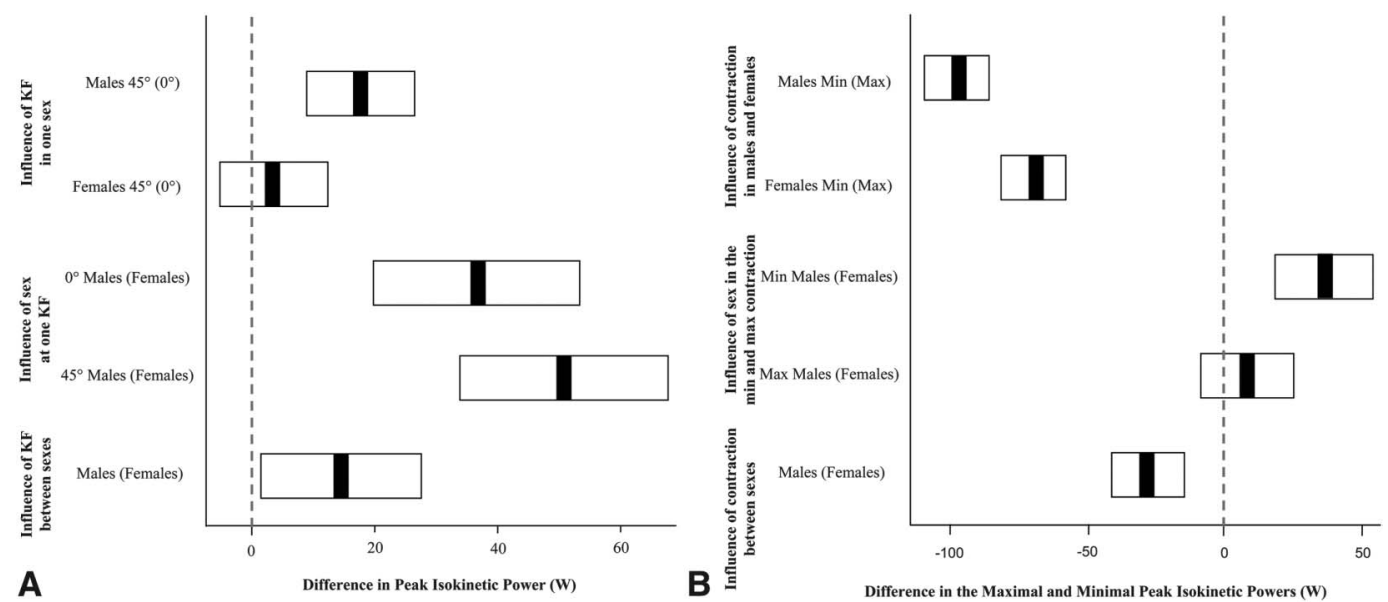

A

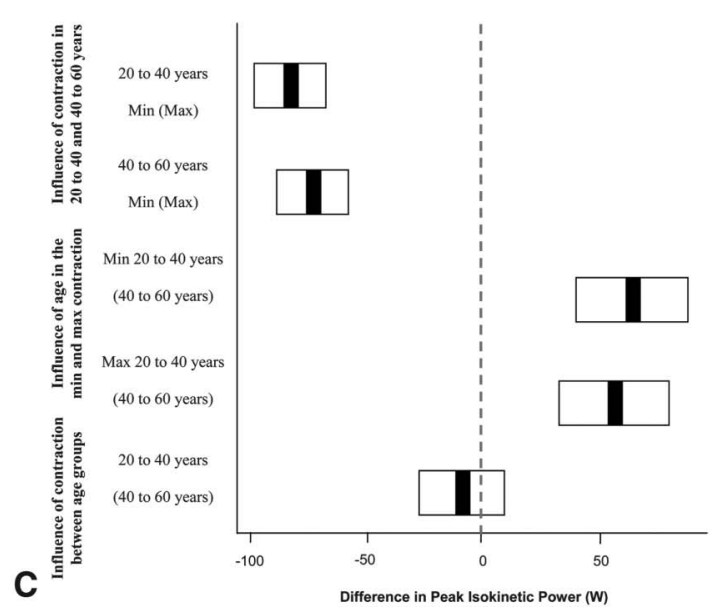

Figure 5. A) Differences in the peak isokinetic power during the maximal and minimal contractions of the 50 concentric plantar flexions of the isokinetic trials in relationship to knee flexion (KF) and sex estimated employing a mixed-effects model. The reference value used for comparison is given in parentheses. The influence of $\mathrm{KF}$ between sexes is the difference in power between the maximal and minimal contractions at angles $0^{\circ}$ angle vs. $45^{\circ}$ angle of $\mathrm{KF}$ in one sex compared with the other. B) Differences in the peak isokinetic power during the maximal and minimal contractions of the 50 concentric plantar flexions of the isokinetic trials in relationship to contraction and sex estimated employing a mixed-effects model. The reference value used for comparison is given in parentheses. The influence of contraction between sexes is the difference in power between the maximal and minimal contractions in one sex compared with the other. C) Differences in the peak isokinetic power during the maximal and minimal contractions of the 50 concentric plantar flexions of the isokinetic trials in relationship to contraction and age group estimated employing a mixed-effects model. The reference value used for comparison is given in parentheses. The influence of contraction between age groups is the difference in power between the maximal and minimal contractions in one age group compared with the other. Comparison (reference), comparison minus reference; represents mean; $\square$ represents $95 \%$ confidence interval; ; represents no difference.

angles $0^{\circ}$ and $45^{\circ}$ of KF. Similarly, the 2-way interaction between the order of testing and the isokinetic contraction $(p=0.4457)$ was removed, indicating a similar decline in powers during the first and second sessions of testing. However, the 2-way interactions between $\mathrm{KF}$ and sex $(p=0.0052$, Figure 5A), sex and isokinetic contraction $(p \leq 0.0001$, Figure $5 \mathrm{~B})$, and age and isokinetic contraction $(p \leq 0.0001$, Figure $5 \mathrm{C})$ were found to influence the peak power.

These interactions showed that the difference between the peak power developed at angles $0^{\circ}$ and $45^{\circ}$ of $\mathrm{KF}$ was greater in males than females $(14 \mathrm{~W}[2,27], p=0.0039)$, as was the difference between the maximal and minimal peak powers of 1 trial $(28 \mathrm{~W}[15,41], p \leq 0.0001)$, even when normalized to body weight. On the other hand, the latter difference in power was similar for the 2 age groups $(8 \mathrm{~W}$ $[-10,27], p=0.2414)$.

Considering the above interactions, the coefficients from the final regressed model provide the following estimates for differences in peak power: 98 [4] W lower for the minimal than maximal peak power during a trial ( $\beta_{\text {contraction, }} p \leq 0.0001$ ), 37 [7] W lower in females than males $\left(\beta_{\text {sex }}, p \leq 0.0001\right), 28[6] \mathrm{W}$ lower in older than younger individuals $\left(\beta_{\text {age }}, p \leq 0.0001\right)$, and $18[4] \mathrm{W}$ lower at angles $0^{\circ}$ than $45^{\circ}$ of KF ( $\left.\beta_{\text {knee, }} p \leq 0.0001\right)$. The order of testing had no significant effect on the peak power output $(p=0.2368)$. 


\section{Discussion}

This investigation was designed to characterize the influence of $\mathrm{KF}$, sex, and age on dynamometric indicators of plantar flexion fatigue from repetitive maximal concentric isokinetic contractions, taking the order of testing into consideration as well. The key finding was that, overall, the KF angle did not significantly alter any of these dynamometric indicators of fatigue (i.e. pre-to-post change in MVIC torque, maximal-tominimal difference in peak isokinetic powers, and normalized slope line of peak isokinetic powers). This result suggests that in endurance-based isokinetic testing, training or rehabilitation of plantar flexion; the differences between the angles $0^{\circ}$ and $45^{\circ}$ of $\mathrm{KF}$ may be too small to discern in clinical settings or of sufficient magnitude to reflect specific responses in the soleus and gastrocnemius muscles. This latter statement does not take into account the differential strains and activation patterns that occur with changes in knee and ankle-joint angles (5) and reflects the fact that kinematic and kinetic parameters are not always adequate indicators of muscle function (12).

Nonetheless, this study identified certain differences that are worth mentioning, such as that $\mathrm{KF}$ exerted a greater impact on the plantar flexion performance of males than females. Another observation here was that although instantaneous measures of plantar flexion performance were higher in younger than older individuals and in males than females, only the male population differed in terms of fatigue indicators. The males exhibited a more pronounced decline in peak isometric and isokinetic contractions than the females, highlighting the importance of taking sex, rather than age, into account when employing and interpreting outcomes from dynamometric assessment procedures similar to those used here. For instance, when attempting to establish whether the values associated with plantar flexion fatigue for a specific individual are within the normal range, it is more important to compare with normative values for the same sex than for a similar age.

Our subjects experienced more pronounced exertion in the triceps surae muscle when the isokinetic trials were performed at angles of $0^{\circ}$ compared with $45^{\circ}$ of $\mathrm{KF}$, despite the fact that the peak isokinetic power was significantly greater at the latter angle. It seems likely that the more intense exertion perceived at the $0^{\circ}$ angle reflects the superior muscle straining of the triceps surae muscles, particularly the gastrocnemius, when the knee is straight (28). At the same time, this small (1-point) difference in the rating of perceived exertion might not be clinically relevant or sufficient to justify inclusion of both $0^{\circ}$ and $45^{\circ}$ angles of $\mathrm{KF}$ in isokinetic protocols involving multiple plantar flexion contractions. This conclusion is supported by our findings of similar dynamometric indicators of fatigue at both knee angles. This lack of any major difference between the angles of $0^{\circ}$ and $45^{\circ}$ may be of significant interest to clinicians and researchers who employ this type of testing, especially over time, because there may be no major concern regarding the exact knee angle of the person being examined.

Most earlier reports show lower absolute plantar flexion strength with the knee bent than straight $(3,5)$, which is in contrast with our estimates of higher isometric torques $(51[3] \mathrm{N} \cdot \mathrm{m})$ and isokinetic powers $(18[4] \mathrm{W})$ at angles $45^{\circ}$ than $0^{\circ}$ of KF. Our higher values are likely to stem from the semi-seated position of the subjects at the $45^{\circ}$ angle, which seemed to promote coactivation of other leg extensors (e.g. the quadriceps), despite the considerable efforts taken to isolate the plantar flexors. Moreover, the position might also have enhanced the inevitable ankle joint rotation reported to occur during maximal plantar flexion efforts in isokinetic dynamometers (4), notwithstanding the means to restrain motion at the ankle. Carlsson et al. (10) also observed higher isometric plantar flexion torques with the knee bent (in sitting) than straight (in prone) that they attributed to plantar flexion being performed in a closed-chain configuration when sitting. We chose to employ an angle of $45^{\circ}$ rather than $90^{\circ}$ because of the higher relevance of the former to daily plantar flexion function. For instance, during the stance phase of running, knee angles of $40^{\circ}-45^{\circ}$ are typical (37). Although in our experimental setup, $45^{\circ}$ angle may have allowed the development of higher plantar flexion forces, the opposite relationship might have been noted at greater $\mathrm{KF}$ angles.

As expected from their relative statures and muscle masses (27), absolute plantar flexion outputs were lower in females than males. Our finding that the males were approximately $45 \%$ stronger than females agrees with the values of $40-50 \%$ reported by Trappe et al. (44) and Danneskiold-Samsøe et al. (13). In general, males are more often involved in strength training activities than females (33), which can influence the ability to activate muscles maximally and generate force (26). However, this factor should have been minimal here because the levels of physical activity were equal in our male and female subjects. Furthermore, Behm and St-Pierre (7) found that resistance-trained compared with -untrained subjects tend to complete a greater number of (sustained isometric) plantar flexion contractions before fatigue, with significantly smaller decrements in MVIC immediately postfatigue. These findings suggest that the stronger males in our current investigation should also demonstrate a greater resistance to fatigue than the weaker females that, however, was not the case.

In fact, our dynamometric indicators of fatigue indicate that our female subjects demonstrated less pre-to-post change in MVIC torque values than the males, reflecting greater resistance to fatigue and more effective maintenance of plantar flexion performance. These findings are consistent with those of Larsson et al. (31), who subjected individuals in their mid-twenties to an isokinetic plantar flexion protocol similar to ours. Biopsies were also taken and the more pronounced and rapid decline in the plantar flexion output of the males were correlated with larger and a higher 
proportion of type II (fast-twitch) muscle fibers. Although the specific mechanisms explaining why the females in our study showed better plantar flexion endurance remain unclear, proposed explanations include disparities in metabolic pathways, activation strategies, muscle mass, perfusion, and hormonal status (15).

With respect to hormonal status, although the findings are mixed, available evidence suggests that estrogen-based treatments exert an overall positive effect on muscle function (19) and that the phase of the menstrual cycle influences the endurance abilities of women (38). We did not control for or obtain precise information concerning such parameters, which is an inherent limitation, Although alterations in the levels of female steroid hormones during the menstrual cycle have been reported to exert no significant impact on the mechanical properties of the medial gastrocnemius muscle and Achilles tendon (30); in rodents, plantar flexion muscle fatigue seems to be attenuated by progesterone in a manner that is counteracted in presence of estradiol (40). Future studies that control for and examine the effect of hormone levels on plantar flexion fatigue in humans using an isokinetic protocol similar to ours are needed to clarify the effect of hormones on plantar flexion performance.

The 3 dynamometric indicators of fatigue that we employed-i.e. the pre-to-post reduction in isometric torque, the difference in power between the maximal and minimal contractions of an isokinetic trial, and the rate of change in peak power during isokinetic trials-were similar for the 2 age groups, despite the fact that human muscles deteriorate in several ways after 40 years of age. Meta-analyses of the effect of aging on muscle function are consistent with our observations (6). Although some evidence suggests that older individuals exhibit greater plantar flexion endurance (34), the latter has only been shown for sustained or intermittent isometric contractions and has not yet been definitively established for dynamic tasks or contractions (6).

In fact, certain evidence suggests that older individuals are less resistant to fatigue than younger ones when performing dynamic contractions at high velocities, but not when contracting at moderate velocities or in static conditions (9). Hence, differences between our age groups might have emerged at higher velocities than the 60 degrees per second employed here. Moreover, it is highly probable that the difference in age between our 2 groups was too small to reveal age-related changes in ankle plantar flexion fatigability. However, when we reanalyzed our data omitting subjects between 35 and 45 years of age not to confound results-thereby comparing subjects between 20 to 35 years to subjects between 45 to 60 years-the findings were identical. Hence, it seems more likely that our older subjects were simply not old enough to detect differences. Applying the current isokinetic protocol to older subjects might reveal greater plantar flexion fatigue with age, as reported by McNeil and Rice (35) for dorsi flexion when comparing young (22-33 years), old (60-69 years), and very old (80-90 years) subjects.
Although the relative performance was similar, absolute plantar flexion outputs were lower in the older age group. In analogy to the discussion of sex differences in plantar flexion function above, the reduction in absolute strength with age is most likely multifactorial. With increasing age, plantar flexion function declines as a consequence of alterations in the cross-sectional areas (36), thickness (17), and contractile tissue volume and distribution (21) of the triceps surae muscles. Although differences in isokinetic plantar flexion performance with age have been reported previously (18), the current findings provide new insights under specific conditions and emphasize that sex is an importance factor to consider when assessing outcomes from repeated concentric isokinetic plantar flexion contractions, e.g. when establishing normative data or comparing individual results.

Another factor to consider when interpreting our current findings is that the subjects were tested in a single experimental session that included familiarization. Although testing on 3 separate occasions ( 1 each for familiarization and isokinetic testing at angles $0^{\circ}$ and $45^{\circ}$ of $\mathrm{KF}$ ) might have been ideal, we do not believe that this would have altered our findings significantly. The order of testing exerted a signifcant impact on the ratings of perceived exertions (i.e. greater exertion was perceived after the second test) and on the absolute MVIC torque values (i.e. both the pre and post values were lower during the second test). However, the pre-to-post change in MVIC torque was not influenced by the order of testing, suggesting similar levels of plantar flexion fatigue. Furthermore, the order of testing had no significant impact on the normalized slopes or difference between the maximal and minimal peak power during isokinetic testing, indicating this order was well controlled for by the randomization and rest periods, with no marked effect of accumulated fatigue on outcomes.

\section{Practical Applications}

Although utilization of $0^{\circ}$ and $45^{\circ}$ of $\mathrm{KF}$ is recommended in connection with a number of clinical procedures for assessing gastrocnemius and soleus function, respectively, the dynamometric indicators of fatigue from concentric plantar flexion contractions performed here at $60^{\circ}$ angle per second were independent of these $2 \mathrm{KF}$ angles. The lack of any major difference between the angles of $0^{\circ}$ and $45^{\circ}$ may be of significant interest to clinicians and researchers who use this test, especially over time, because there may be no major concern regarding the exact knee angle of the individual being tested. Moreover, although instantaneous peak isometric torque and isokinetic power were lower in the subjects older than 40 years and in female subjects, there were no age-related differences in the dynamometric indicators of fatigue or rate of change in plantar flexion performance. On the other hand, the males in our study exhibited a more pronounced reduction in peak isometric torque and isokinetic power than the females. Therefore, clinicians and scientists are encouraged to consider sex, rather than age, when 
designing and interpreting results from isokinetic tests of plantar flexion endurance, e.g. when determining normative values and comparing these to pathological outcomes. Future studies that examine the impact of hormone levels and extreme age on the dynamometric indicators of plantar flexion fatigue focused on here would help clarify the influence of $\mathrm{KF}$ angle, sex, and age on muscular fatigue.

\section{ACKNowledgments}

The authors acknowledge the research assistance from Sarah Willis, MSc, and Maria Hansson, PT, during the data collection. We also like to thank all subjects who volunteered to take part in this study.

\section{REFERENCES}

1. Alfredson, H, Pietila, T, Jonsson, P, and Lorentzon, R. Heavy-load eccentric calf muscle training for the treatment of chronic Achilles tendinosis. Am J Sports Med 26: 360-366, 1998.

2. Andersen, $\mathrm{H}$ and Jakobsen, J. A comparative study of isokinetic dynamometry and manual muscle testing of ankle dorsal and plantar flexors and knee extensors and flexors. Eur Neurol 37: 239-242, 1997.

3. Arampatzis, A, Karamanidis, K, Stafilidis, S, Morey-Klapsing, G, DeMonte, G, and Brüggemann, GP. Effect of different ankle- and knee-joint positions on gastrocnemius medialis fascicle length and EMG activity during isometric plantar flexion. $J$ Biomech 39: 18911902, 2006.

4. Arampatzis, A, Morey-Klapsing, G, Karamanidis, K, DeMonte, G, Stafilidis, S, and Brüggemann, GP. Differences between measured and resultant joint moments during isometric contractions at the ankle joint. J Biomech 38: 885-892, 2005.

5. Arndt, AN, Komi, PV, Brüggemann, GP, and Lukkariniemi, J. Individual muscle contributions to the in vivo Achilles tendon force Clin Biomech (Bristol, Avon) 13: 532-541, 1998.

6. Avin, KG and Frey Law, LA. Age-related differences in muscle fatigue vary by contraction type: A meta-analysis. Phys Ther 91 : 1153-1165, 2011.

7. Behm, DG and St-Pierre, DMM. Fatigue mechanisms in trained and untrained plantar flexors. J Strength Cond Res 12: 166-172, 1998.

8. Bretz, F, Hothorn, T, and Westfall, P. Multiple Comparisons Using $R$. Boca Rato, FL: Taylor and Francis Group, 2010.

9. Callahan, DM and Kent-Braun, JA. Effect of old age on human skeletal muscle force-velocity and fatigue properties. J Appl Physiol 111: 1345-1352, 2011.

10. Carlsson, U, Lind, K, Möller, M, Karlsson, J, and Svantesson, U. Plantar flexor muscle function in open and closed chain. Clin Physiol 21: 1-8, 2001.

11. Craig, CL, Marshall, AL, Sjöström, M, Bauman, AE, Booth, ML, Ainsworth, BE, Pratt, M, Ekelund, ULF, Yngve, A, Sallis, JF, and Oja, P. International Physical activity questionnaire: 12-country reliability and validity. Med Sci Sports Exerc 35: 1381-1395, 2003.

12. Cronin, NJ, Prilutsky, BI, Lichtwark, GA, and Maas, H. Does ankle joint power reflect type of muscle action of soleus and gastrocnemius during walking in cats and humans? J Biomech 46: 1383-1386, 2013.

13. Danneskiold-Samsøe, B, Bartels, EM, Bülow, PM, Lund, H, Stockmarr, A, Holm, CC, Wätjen, I, Appleyard, M, and Bliddal, H. Isokinetic and isometric muscle strength in a healthy population with special reference to age and gender. Acta Physiol (Oxf) 197: 1-68, 2009.

14. Emery, CA. Injury prevention and future research. Med Sport Sci 48: 179-200, 2005

15. Enoka, RM and Duchateau, J. Muscle fatigue: What, why and how it influences muscle function. $J$ Physiol 586: 11-23, 2008.
16. Ferreira, ML, Sherrington, C, Smith, K, Carswell, P, Bell, R, Bell, M, Nascimento, DP, Máximo Pereira, LS, and Vardon, P. Physical activity improves strength, balance and endurance in adults aged 40-65 years: A systematic review. J Physiother 58: 145-156, 2012.

17. Fujiwara, K, Asai, H, Toyama, H, Kunita, K, Yaguchi, C, Kiyota, N, Tomita, $\mathrm{H}$, and Jacobs, JV. Changes in muscle thickness of gastrocnemius and soleus associated with age and sex. Aging Clin Exp Res 22: 24-30, 2010.

18. Gajdosik, RL, Vander Linden, DW, and Williams, AK. Concentric isokinetic torque characteristics of the calf muscles of active women aged 20 to 84 years. J Orthop Sports Phys Ther 29: 181-190, 1999.

19. Greising, SM, Baltgalvis, KA, Lowe, DA, and Warren, GL. Hormone therapy and skeletal muscle strength: A meta-analysis. $J$ Gerontol $A$ Biol Sci Med Sci 64A: 1071-1081, 2009.

20. Harbo, T, Brincks, J, and Andersen, H. Maximal isokinetic and isometric muscle strength of major muscle groups related to age, body mass, height, and sex in 178 healthy subjects. Eur J Appl Physiol 112: 267-275, 2012.

21. Hasson, CJ, Kent-Braun, JA, and Caldwell, GE. Contractile and noncontractile tissue volume and distribution in ankle muscles of young and older adults. $J$ Biomech 44: 2299-2306, 2011.

22. Hébert-Losier, K, Schneiders, AG, Garcia, JA, Sullivan, SJ, and Simoneau, GG. Influence of knee flexion angle and age on triceps surae muscle fatigue during heel raises. J Strength Cond Res 26: 31343147, 2012.

23. Hébert-Losier, K, Schneiders, AG, Sullivan, SJ, Newsham-West, RJ, García, JA, and Simoneau, GG. Analysis of knee flexion angles during two clinical versions of the heel-raise test to assess soleus and gastrocnemius function. J Orthop Sports Phys Ther 41: 505-513, 2011.

24. Heckman, DS, Gluck, GS, and Parekh, SG. Tendon disorders of the foot and ankle, part 2: Achilles tendon disorders. Am J Sports Med 37: 1223-1234, 2009.

25. Hislop, $\mathrm{HJ}$ and Montgomery, J. Daniels and Worthingham's Muscle Testing: Techniques of Manual Examination. W.B. Saunders, 8th edition. St. Louis, MO: Saunders, Elsevier Inc., 2007.

26. Hortobágyi, T, Barrier, J, Beard, D, Braspennincx, J, Koens, P, Devita, P, Dempsey, L, and Lambert, J. Greater initial adaptations to submaximal muscle lengthening than maximal shortening. $J$ Appl Physiol 81: 1677-1682, 1996.

27. Janssen, I, Heymsfield, SB, Wang, Z, and Ross, R. Skeletal muscle mass and distribution in 468 men and women aged 18-88 yr. J Appl Physiol 89: 81-88, 2000.

28. Kawakami, Y, Ichinose, Y, and Fukunaga, T. Architectural and functional features of human triceps surae muscles during contraction. J Appl Physiol 85: 398-404, 1998.

29. Kendall, FP, McCreary, EK, Provance, PG, Rodgers, MM, and Romani, WA. Muscles: Testing and Function With Posture and Pain. Baltimore, MD: Lippincott Williams and Wilkins, 2010.

30. Kubo, K, Miyamoto, M, Tanaka, S, Maki, A, Tsunoda, N, and Kanehisa, H. Muscle and tendon properties during menstrual cycle. Int J Sports Med 30: 139-143, 2009.

31. Larsson, B, Kadi, F, Lindvall, B, and Gerdle, B. Surface electromyography and peak torque of repetitive maximum isokinetic plantar flexions in relation to aspects of muscle morphology. J Electromyogr Kinesiol 16: 281-290, 2006.

32. Larsson, B, Månsson, B, Karlberg, C, Syvertsson, P, Elert, J, and Gerdle, B. Reproducibility of surface EMG variables and peak torque during three sets of ten dynamic contractions. J Electromyogr Kinesiol 9: 351-357, 1999.

33. Loustalot, F, Carlson, SA, Kruger, J, Buchner, DM, and Fulton, JE. Muscle-strengthening activities and participation among adults in the United States. Res Q Exerc Sport 84: 30-38, 2013.

34. Mademli, L and Arampatzis, A. Mechanical and morphological properties of the triceps surae muscle-tendon unit in old and young adults and their interaction with a submaximal fatiguing contraction. J Electromyogr Kinesiol 18: 89-98, 2008. 
35. McNeil, CJ and Rice, CL. Fatigability is increased with age during velocity-dependent contractions of the dorsiflexors. J Gerontol A Biol Sci Med Sci 62: 624-629, 2007.

36. Morse, CI, Thom, JM, Davis, MG, Fox, KR, Birch, KM, and Narici, MV Reduced plantarflexor specific torque in the elderly is associated with a lower activation capacity. Eur J Appl Physiol 92: 219-226, 2004.

37. Novacheck, TF. The biomechanics of running. Gait Posture 7: 77-95, 1998.

38. Oosthuyse, T and Bosch, AN. The effect of the menstrual cycle on exercise metabolism: implications for exercise performance in eumenorrhoeic women. Sports Med 40: 207-227, 2010.

39. Ritti-Dias, RM, Basyches, M, Câmara, L, Puech-Leao, P, Battistella, L, and Wolosker, N. Test-retest reliability of isokinetic strength and endurance tests in patients with intermittent claudication. Vasc Med 15: 275-278, 2010.

40. Schneider, BSP, Fine, JP, Nadolski, T, and Tiidus, PM. The effects of estradiol and progesterone on plantarflexor muscle fatigue in ovariectomized mice. Biol Res Nurs 5: 265-275, 2004.

41. Schneiders, AG, Sullivan, SJ, O’Malley, KJ, Clarke, SV, Knappstein, SA, and Taylor, LJ. A valid and reliable clinical determination of footedness. PM R 2: 835-841, 2010.

42. Shephard, RJ. Aging and exercise. In: Encyclopedia of Sports Medicine and Science. T Fahey, ed. Internet Society for Sport Science. Available at: http://sportsci.org, 1998. Accessed June 6, 2007.

43. Signorile, JE, Applegate, B, Duque, M, Cole, N, and Zink, A. Selective recruitment of the triceps surae muscles with changes in knee angle. J Strength Cond Res 16: 433-439, 2002.

44. Trappe, SW, Trappe, TA, Lee, GA, and Costill, DL. Calf muscle strength in humans. Int J Sports Med 22: 186-191, 2001.

45. Westerblad, H and Allen, DG. Changes of myoplasmic calcium concentration during fatigue in single mouse muscle fibers. $J$ Gen Physiol 98: 615-635, 1991. 